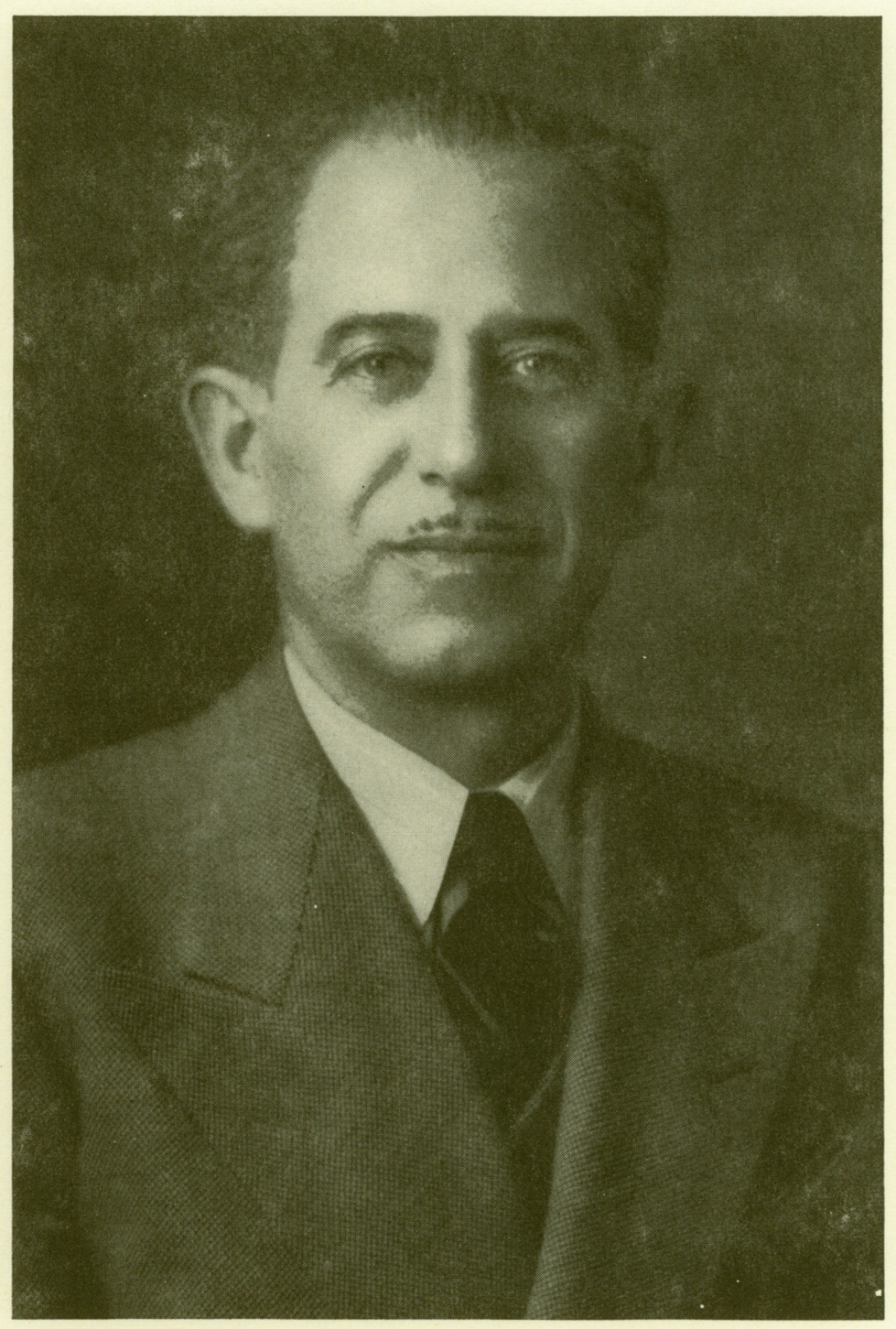

A. N. LOWAN 1945 


\section{The Solution of Simultaneous Linear Equations with the Aid of the 602 Calculating Punch}

I. Introduction.-The method of solving simultaneous equations described here is the basic elimination method with certain modifications and improvements. Specifically, the usual elimination method requires the use of a "back-substitution" procedure to evaluate the remaining $(n-1)$ unknowns after the $n$th unknown is determined. This "back-substitution" procedure is a departure from the basic elimination formula and requires a change in the elimination technique. In the proposed method "backsubstitution" is eliminated, and the same basic elimination procedure is repeated throughout. After $n$ successive reductions, one obtains the values of the $n$ unknowns on $n$ corresponding punched cards.

This improvement is effected by the use of an augmented matrix of somewhat greater dimensions. The character of the particular augmented matrix is determined by the desired end result. In certain cases it is necessary to obtain only the values of the respective unknowns. Other physical problems are such that the matrix of the coefficients of the unknowns remains invariant, whereas the matrix of the constant terms is varied and several solutions are required for the given coefficient matrix. Finally, in some cases it is desirable to compute the inverse of the given matrix of the coefficients of the unknowns. The corresponding variations in the composite matrix for each of these three cases is given in the following mathematical discussion.

The choice of this modified elimination method as the basic scheme to be mechanized was further dictated by its direct nature. In contrast to the indirect iteration procedures, only one application of the method is necessary to obtain the desired solutions with an accuracy consistent with the number of significant figures in the given matrices and the associated round-off error incurred. There is never a question of the lack of convergence of the method or even the slowness of convergence of the method.

Much has been written regarding the magnitude of the round-off errors incurred in such an elimination procedure. ${ }^{1,2,3}$ Since these derivations of the magnitude of the possible round-off errors are based on maximum value criteria, they are very pessimistic. Fortunately, in most practical cases encountered, this error is small. The retention of one, or at the most two, extra guard figures is frequently an adequate precaution against the accumulation of round-off errors. ${ }^{4}$

II. Mathematical Discussion.-Consider the set of simultaneous linear equations,

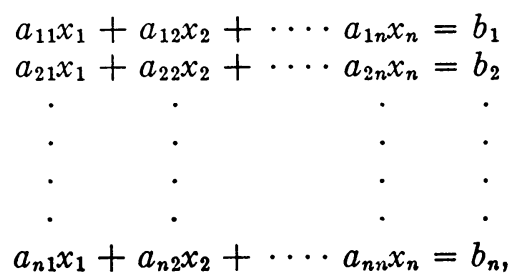


which may be rewritten in matrix notation as

$$
A X=B \text {, }
$$

in which $A$ is a matrix of order $(n \times n)$ and $X$ and $B$ are single column matrices representing vectors of the $n$th order.

In the usual elimination method, the equations are so arranged that $a_{11}$ is not zero and the first equation is divided by $a_{11}$. By successively subtracting $a_{k 1}$ (where $a_{k 1}$ represent the leading column coefficients of the last $(n-1)$ equations) times this new equation from each of succeeding $(n-1)$ equations, $x_{1}$ is eliminated from these $(n-1)$ equations. If the resulting $(n-1)$ square matrix is similarly treated, $x_{2}$ will be eliminated from the last $(n-2)$ equations. After $(n-1)$ such reductions, we have a single equation relating the last unknown and a constant term, i.e.,

$$
h_{n n} x_{n}=c_{n},
$$

which may be solved for $x_{n}$. This value of $x_{n}$ may be substituted back into the next-to-the-last equation relating $x_{n-1}$ and $x_{n}$, and the value of $x_{n-1}$ obtained. In a similar manner the remaining unknowns, $x_{n-2}, x_{n-3}, \cdots x_{2}, x_{1}$, may be evaluated by "back-substitution."

This "back-substitution" procedure may be avoided by the use of the following composite matrix:

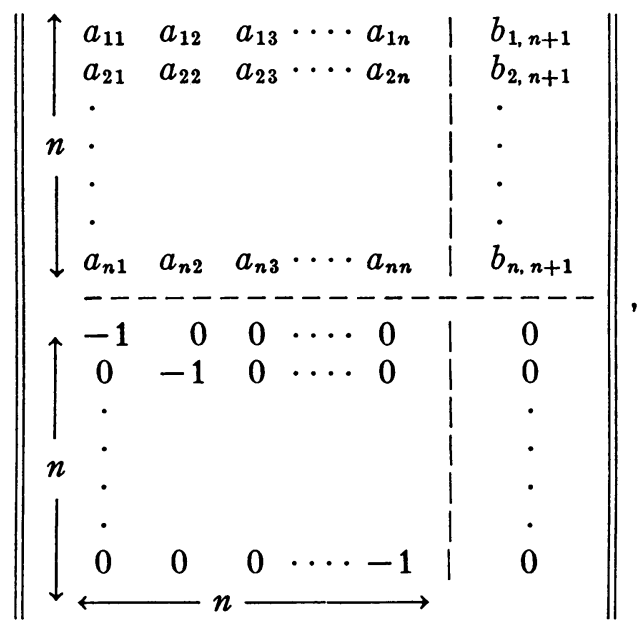

which may be rewritten in matrix notation as:

$$
\begin{array}{c|c}
A & B \\
\hdashline-I & O
\end{array}
$$

If the same basic forward elimination operations are applied to this composite matrix for $n$ successive reductions (see later discussion of Machine Computation for exact details of these operations), we obtain a matrix of 
the form:

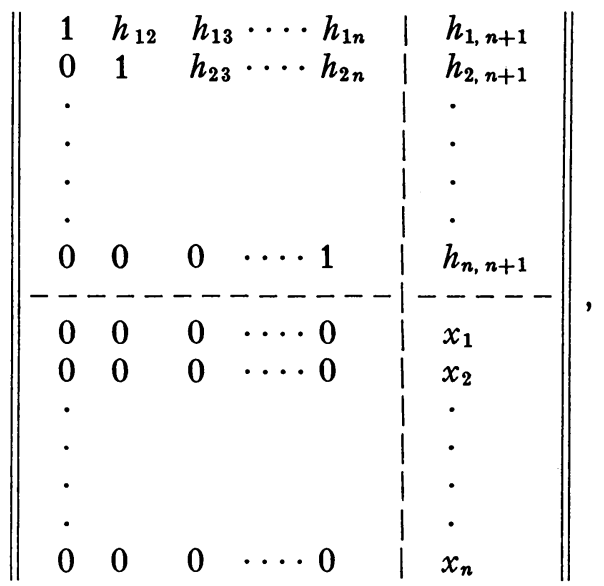

in which the desired $n$ unknowns appear in column $(n+1)$ without the use of the "back-substitution" procedure.

A further consideration of the matrices of (1) and (2) will reveal that certain terms of the negative identity matrix $-I$ contribute nothing during the above elimination process. These terms are those below the main diagonal. Therefore, the "unit upper triangular matrix" may be used with an economy of space and an increase in speed of operation.

In certain physical applications sets of simultaneous equations occur in which the coefficient matrix $A$ is a constant but the constant term matrix $B$ assumes different values for each set of equations. Clearly, such a system of equations is directly amenable to solution. In fact, the entire group of $m$ sets may be simultaneously evaluated with an identical procedure using the following composite matrix:

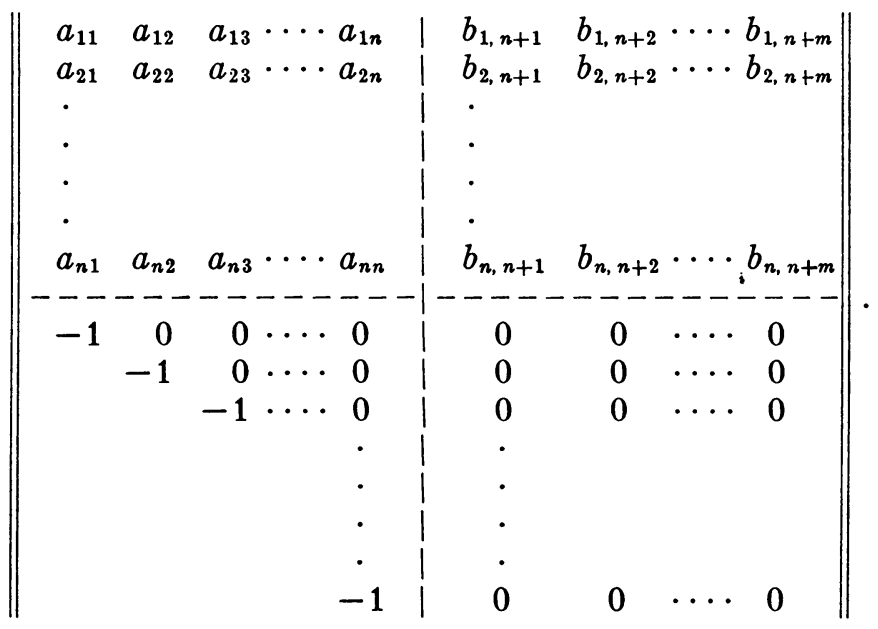

This matrix resembles (1) except that additional columns have been added to both the $B$ and $O$ matrices. The reduction procedure is identical with that described above except that $2 n(m-1)$ additional cards are needed. 
The evaluation of the inverse of a matrix may be readily accomplished by this method. The decision as to whether a direct solution is to be obtained or whether the inverse matrix is to be evaluated depends upon the physical problem represented as well as the relative magnitudes of $m$ and $n$.

The inverse matrix provides an accurate indication of the sensitivity of the solution to small changes in the coefficients of $A$ and $B$. In addition, the unknowns may be readily evaluated for various values of $B$ by means of the following equation:

$$
X=A^{-1} B \text {. }
$$

The actual number of multiplications involved in this matrix multiplication is determined by the respective values of $m$ and $n$. It is sometimes advisable to solve the following composite matrix:

$$
\| \begin{array}{c|c|c}
A & B & I \\
\hdashline-I & O & O
\end{array} \mid .
$$

If the same basic elimination procedure is followed, after $n$ reductions the values of the unknowns will appear in the column of the original $O$ matrix located under the $B$ matrix. Furthermore, the inverse matrix $A^{-1}$ will appear in the original $(n \times n) O$ matrix located under the $I$ matrix.

The $B$ matrix and its associated single-column $O$ matrix may be omitted from (3), and only the inverse of $A$ will be obtained. However, the inclusion of the $B$ matrix entails very little additional work, and it does provide an immediate check on the accuracy obtained. That is, the values of the unknowns for one $B$ are available, and they may be substituted into the original equations, and the residuals ascertained.

Sometimes a set of simultaneous equations is obtained with a single column entry for $B$, it being known however that many different values of $B$ will arise in the course of the experimental investigation for a constant value of $A$. In such a case it is advisable to evaluate (3) in the form given and use the value of the inverse of $A$ to evaluate the unknowns for each new value of $B$ as it occurs in the later investigations.

III. Machine Computation with the Aid of the 602 Calculating Punch. -At any stage of the reduction of the composite matrix

$$
G=\left\|\begin{array}{c|c}
A_{n n} & B_{n 1} \\
\hdashline-I_{n n} & O_{n 1}
\end{array}\right\|,
$$

only $(n+1)$ rows of the matrix are used because of the character of the $-I$ matrix. That is, the leading column coefficient of the $(n+2)^{\text {th }}$ row is already zero. At each succeeding reduction, one leading row of the composite matrix is omitted and a new row is added-maintaining $(n+1)$ rows throughout the solution. The number of columns to the left of the vertical line is reduced by one at each reduction. Finally, after the $n$th reduction, nothing remains to the left of the vertical line, and the desired unknowns appear in the lower right-hand corner. 
Punched-Card Layout.-In addition to the actual coefficients which appear in the above matrix some identification of data is necessary to permit efficient machine operation. The following punched-card layout is used:

\begin{tabular}{cl} 
Card Columns & \multicolumn{1}{c}{ Data } \\
$1-2$ & row number of element in $G$ \\
$3-4$ & column number of element in $G$ \\
5 & 1 for elements of $A$ \\
& 2 for elements of $-I$ \\
& 3 for elements of $B$ \\
& 4 for elements of $O$ \\
& 5 for elements of $I$ \\
& 6 for elements of $O^{+}$ \\
$6-7$ & reduction number \\
$8-10$ & problem number \\
$11-20$ & sign and data $\left(P=a_{i j}\right)$ \\
& (The decimal point appears between columns 13-14 when \\
& seven-decimal places are used in the computation.) \\
$21-30$ & value of $Q=a_{i 1}(\operatorname{sign}$ in column 22$)$ \\
$31-40$ & value of $R=\left(a_{1 i} / a_{11}\right)($ sign in column 31$)$ \\
$44-53$ & value of $\left\|a_{i j}-a_{i 1}\left(a_{1 j} / a_{11}\right)\right\|=\|P-Q R\|$.
\end{tabular}

Key Punching and Verification Procedure.-The respective coefficients of the $A$ and $B$ matrices are supplied to the Key Punch operator on a special coefficient form sheet. In addition to the actual columns of coefficients there is a check column supplied, each entry of which is the sum of all the elements in its corresponding row. Similarly, a check row is supplied, each entry of which in turn is a sum of all the elements in its corresponding column. These check vectors serve two purposes: first, as a check of the key-punched data; second, as a continuous check throughout the actual computation.

The verification of the key punching is effected by means of a suitably wired tabulator board. Each coefficient of the composite matrix is entered on a separate punched card in card columns 11 through 20. The tabulator board is wired to print the successive coefficients of a particular row on the same horizontal line in a tabular array in accordance with the columns of the given matrix. Furthermore, the board is wired to obtain the totals of the columns. It is evident that one can automatically check the key punching of all entries by passing the cards through the tabulator twice-once with the cards arranged in order of matrix row and next with the cards arranged in order of matrix column. Any discrepancy between the known check sums will affect two check sums and immediately identify the entry which is in error.

Flow Chart for the 602 Calculating Punch.-The basic elimination process consists of evaluating the following mathematical expression:

$$
\|P-Q R\|=\left\|a_{i j}-a_{i 1}\left(a_{1 j} / a_{11}\right)\right\|,
$$

in which the use of subscripts will be avoided hereafter by use of the letters $P, Q$, and $R$. Because of the repetitive nature of this reduction process, the quantity $(P-Q R)$ of one reduction becomes the $P$ of the next reduction. The actual manner in which this is effected may be seen from Figure 1.

Division Cards.- The coefficients of the leading row of the matrix are used to evaluate $R$ in accordance with the equation:

$$
R=a_{1 j} / a_{11}=D / E .
$$




\begin{tabular}{|c|c|c|c|c|c|c|c|c|c|c|c|}
\hline \multirow{2}{*}{$\begin{array}{l}\text { Ouetomer: } \\
\text { D1souselo } \\
\end{array}$} & \multirow{2}{*}{$\begin{array}{c}\text { IOAND } \\
\text { D21180r } \\
3=4\end{array}$} & \multirow{2}{*}{$\begin{array}{l}\text { IER } \\
\text { Quot. } \\
1-2\end{array}$} & \multicolumn{2}{|c|}{ LHC } & \multicolumn{2}{|c|}{ R月O } & \multicolumn{2}{|c|}{ Bummasy } & \multicolumn{3}{|c|}{ Rosult Storage } \\
\hline & & & $\begin{array}{l}9^{\text {D12 }} \\
9\end{array}$ & $\begin{array}{c}\text { aend } \\
10-11\end{array}$ & $5-8$ & $6-7$ & 13-16 & $14-15$ & & & \\
\hline & & & Ro & & & & $\mathrm{RC}$ & & & & \\
\hline $\begin{array}{c}\text { Road } \\
\text { Riv1 Bor } \\
x>2\end{array}$ & Add & & $\underset{\mathrm{D}}{\mathcal{A d A d}}$ & & & & & \begin{tabular}{|c} 
Add \\
Iáen.
\end{tabular} & & & \\
\hline Prog CII 3 & 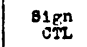 & & & & & & & & & & \\
\hline Prog CTL 4 & & & 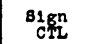 & & & & & & & & \\
\hline Divide & $\mathrm{RC}$ & $R=\frac{D}{E}$ & & & & & & & & & \\
\hline $\begin{array}{l}\text { TRAHSFER } \\
\text { STOROAE }\end{array}$ & & Ro-RC & RC & & & & $\underset{\mathbf{R}}{\operatorname{Add}}$ & & & & $\begin{array}{l}\text { Punch } \\
\text { R }\end{array}$ \\
\hline $\begin{array}{c}\text { Read } \\
\text { Detetal1 } \\
x \in 8\end{array}$ & $\stackrel{A d d}{Q}$ & & subt $\bar{p}$ & & & & & Add & & & \\
\hline Prog $\mathrm{CTL} 1$ & $\begin{array}{l}31 \mathrm{gn} \\
\mathrm{CZI}\end{array}$ & $\begin{array}{l}\text { Add- } \\
\text { SuUt R } \\
\text { s1gn }\end{array}$ & & & $\begin{array}{c}\text { Subt } \\
\begin{array}{c}\text { LAd } \\
\mathrm{E} / 2\end{array}\end{array}$ & $\begin{array}{c}\text { Provide } \\
\mathbf{E} / 2\end{array}$ & $\begin{array}{c}\mathrm{Bal} \\
\mathrm{RC}\end{array}$ & & & & \\
\hline $\begin{array}{l}\text { Negntivo } \\
\text { Sultipiy }\end{array}$ & Re & RC & $(8-8 R)$ & & $\mathrm{RC}$ & & & & & & \\
\hline $\begin{array}{c}\text { TRAHSFTR } \\
\text { TO } \\
\text { STORACE }\end{array}$ & & & so & & & & Ro & & & & \\
\hline & & & & & & & & & $(8-a)$ & & $\mathrm{R}$ \\
\hline $\begin{array}{c}\text { Read } \\
\text { BLANK } \\
\text { CARD }\end{array}$ & & & & & & & & $\underset{1}{A d d}$ & & & \\
\hline $\begin{array}{c}\text { TRANGFSR } \\
\text { I0 } \\
\text { STORAGE }\end{array}$ & RC & & $\mathrm{RO}-\mathrm{RC}$ & & RC & & & RO-RC & $(P-R R)$ & $\begin{array}{l}\text { Ident } \\
\text { Iloat 1op }\end{array}$ & \\
\hline
\end{tabular}

Fig. 1. Flow Chart Diagram of the 602 Calculating Punch.

The punched cards corresponding to the leading row are characteristically designated by an X71 punch. The values of $D$ and $E$ are added into the dividend and divisor counters of the 602, respectively. Since these quantities may be either positive or negative, the algebraic sign must be examined, and the correct sign of the quotient $R$ is determined automatically by the algebraic sign control circuits of the 602 calculator. The quotient is punched on the card and is stored within the calculator for use in succeeding computations.

Multiplication Cards. - The remaining cards of the matrix (exclusive of the leading row X71 cards) are designated by an X68 punch. Each of these cards contains its corresponding matrix coefficient, $P=a_{i j}$, as well as the associated element in the leading column, i.e., $Q=a_{i 1}$. Since the entire set of cards corresponding to the composite matrix are sorted in order of the 
matrix column in which they appear, all X68 cards of a particular column follow the appropriate X71 card.

The values of $P$ and $Q$ are read into the multiplicand and $L H C$ (left hand component) counter, respectively, for each X68 card. The calculator uses the previously stored value of $R$ as a multiplier and performs a combined crossfooting and negative multiplication operation to obtain $P-Q R$ with due regard to the algebraic sign of the respective variables. Notice that $R$ is used as a group multiplier for all cards of a particular column of the matrix.

The quantities $(P-Q R)$ and $R$ are punched on each $\mathrm{X} 68$ card. The value of $R$ is required whenever the product $Q R$ is verified by the calculator in a verification-of-multiplication operation. The identification in card columns $1-10$ is read into counters $14-15$ for both X68 and X71 cards and is retained there until it is transferred to the appropriate columns of the following blank card.

Blank Trailer Cards.-Each of the X71 and X68 cards is followed by a blank trailer card. These blank cards receive their appropriate identification by reading out the contents of counters 14-15 into card columns $1-10$. They also have the value of $P-Q R$ punched upon them in card columns 11-20, i.e., the quantity $(P-Q R)$ of reduction no. 1 becomes the new $P$ for reduction no. 2, etc. In effect the 602 performs an interspersed offset-gang-punch operation. Furthermore, the blank cards have the corrected reduction number punched in card columns 6-7. The set of "blank" cards of one reduction becomes the new active set in the next reduction.

Operational Steps in a Reduction Process.-The following reduction procedure is equally applicable whether the operation is:

a. the evaluation of the unknowns for a single system of equations,

b. the concurrent evaluation of the unknowns for several sets of simultaneous equations,

c. the evaluation of the unknowns for a constant $A$ matrix and a multiple column $B$ matrix system of equations,

d. the evaluation of the inverse of a matrix, $A^{-1}$.

The only difference is the respective constituents of the original composite matrix. Specifically, the matrix (4) is used in the solution of $n$ simultaneous equations. The procedure is as follows: ${ }^{5}$

1. Select the first $(n+1)$ rows of the above composite matrix (i.e., all the rows of $A$ and $B$ plus the first row of $-I$ and $O$ ). Sort on card columns 2,1 .

2. Sort on card columns 4,3 , and remove the leading column cards from the above selected group.

3. Gang-punch X71, X77, and X80 on the first card of this leading column group. Gang-punch X68, X77, and X80 on the remaining cards of this leading column group.

4. Reproduce a copy of these leading column cards on yellow-top cards EXCEPT for the $\mathrm{X} 77$ and $\mathrm{X} 80$.

5. Assemble the cards in the following order:

a) X80 cards of the leading column group,

b) NX80 cards (i.e., cards which do not have an X in position 80) of the leading column group,

c) remnants of step 2 .

6. Sort the assembled group in order of increasing matrix row number, i.e., card columns $2,1$. 
7. Offset-gang-punch " $Q$ " from the leading column $\mathrm{X} 80$ cards upon the other cards of the matrix in the SAME row. (X68 and X71 are gang-punched directly).

8. Verify the offset-gang-punching.

9. Sort on column 80 , remove, and file the $\mathrm{X} 80$ cards.

10. Sort on card columns 4,3 , and arrange the cards in order of matrix column.

11. Insert a blank trailer card after each card of this group.

12. Card count this merged set, and check the card count against the supplied check data sheet.

13. Precede this new group of cards with a blank card, and feed the entire group to the 602.

14. Sort the cards obtained from the 602 on card column 7, and segregate the former "blank" cards from the original composite matrix. (Label and file the latter.)

15. Sort on card columns 4,3 , and remove and file the leading column cards of the new matrix (i.e., the former blank cards). These are all equal to zero.

16. Check the computation in this reduction by feeding these cards to the tabulator. The sum of the entries in a particular row is automatically obtained by the tabulator, and it should agree with the number on the card of the final check column.

17. Sort on card column 2, 1 , and remove and file the leading row cards of the new matrix. Again, these should all be zero.

18. Add the $(n+2)^{\text {th }}$ row of the original composite matrix to the new matrix (i.e., add the second row of $-I$ and $O$ to the new matrix). Check the card count sheet.

19. Repeat steps 2 to $18 n$ times.

The cyclic nature of this elimination procedure is clearly shown in Figure 2. The numbers next to the machine diagram refer to the corresponding step in the above reduction procedure. Each reduction is effected by a single passage of the cards around the circular path 2 to 18 .

Checking Procedures.-Several of the operations shown in Figure 2 serve only as a check on the reduction procedure. These checks include:

1. an automatic verification of the offset-gang-punching,

2. a continuous check on card count,

3. a verification of the 602 computation of $S=(P-Q R)$ by automatically checking the following equation:

for a zero balance,

$$
S-(P-Q R)=0
$$

4. a computational check by means of the inclusion of check row and check column cards.

An additional final check may be performed, although it is not shown on the flow chart diagram. This check consists of an evaluation of the residuals which are obtained when the answers are substituted back into the original equations. This check provides an accurate evaluation of the magnitude of the round-off error which is incurred during the elimination process.

Evaluation of the Residuals.-The $n$ cards which are obtained as a result of the $n$th reduction have the values of the $n$ unknowns in card columns 11-20. If this set of cards is reproduced reversing the row and column identification, we obtain $x_{1}$ in column $1, x_{2}$ in column 2, etc. If an X71, X22, and a 1 in column 23 are gang-punched on each of these cards, they may be used to evaluate the residuals with the same 602 and tabulator boards.

These cards served as "divisor" cards in which the unknown is divided by -1 to obtain the necessary sign reversal in the expression $(P-Q R)$. In this case $P=0$ and $R$ represents the unknowns $x_{n}$. The variable $Q$ represents the given coefficients $a_{i j}$, i.e., in effect we solve the equation

$$
(P-Q R)=0-a_{i j}\left(-x_{n}\right)=a_{i j} x_{n} .
$$




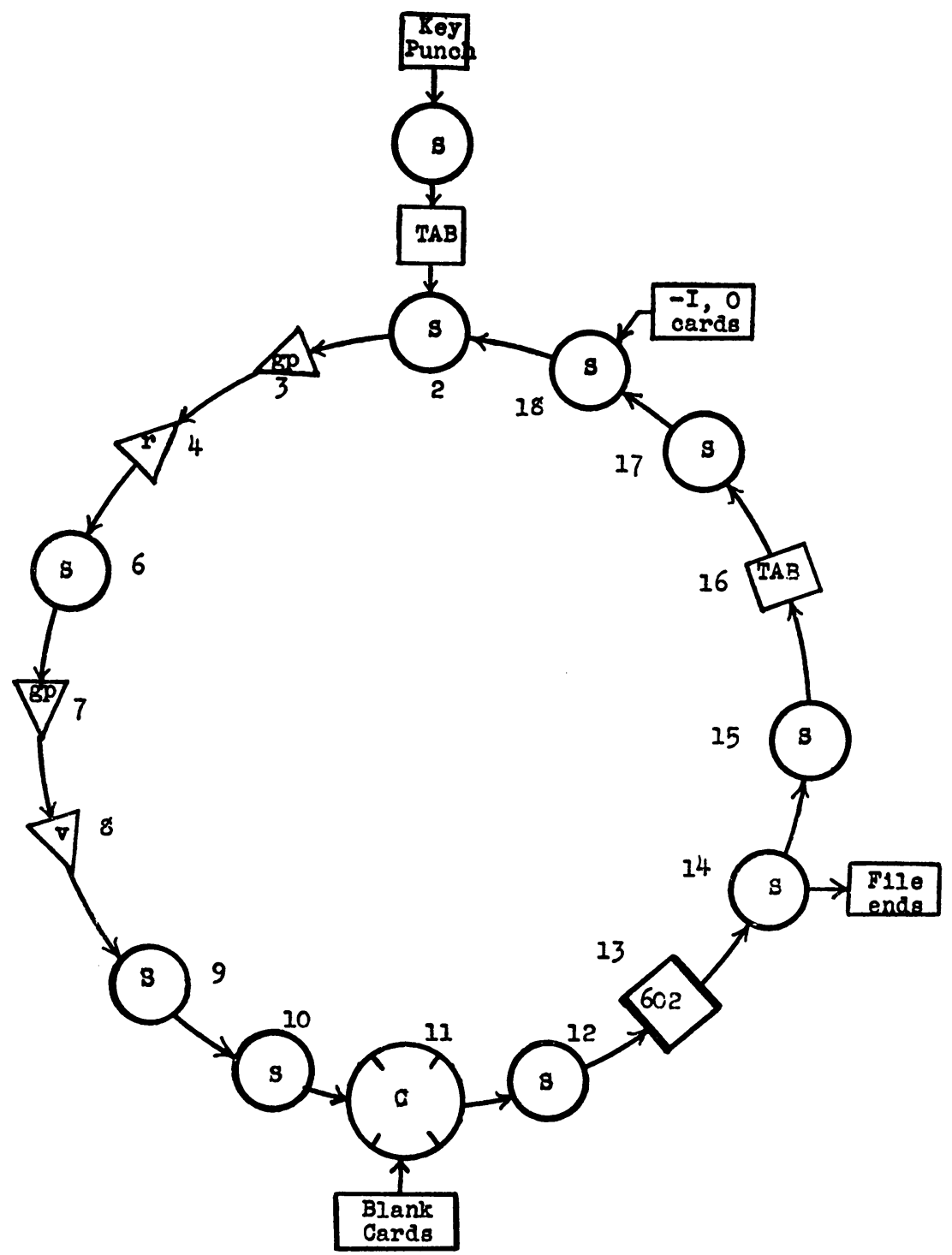

Fig. 2. A Machine Flow Chart Diagram of the Elimination Method.

Hence, by sorting the original cards of matrix $A$ behind the associated $x_{n}$ card in order of matrix column, the 602 utilizes the particular $x_{n}$ as a group multiplier and provides the desired product $a_{i j} x_{n}$ on each card. If these cards are now fed to the tabulator which totals the cards on matrix row number, we obtain a sum which is the desired residual.

Accuracy Considerations. - The final results are obviously in error by an amount which is determined by the rounding-off errors. One or two guard figures are required in the original coefficient to insure the desired accuracy 
in the final answers. The exact magnitude of the error incurred in the case of a set of ill-conditioned equations is still subject to question and requires additional investigation with the aid of high-speed digital equipment.

The usual methods for shifting the decimal point of the given matrix so that the maximum coefficient is near unity are easily effected in this procedure. Furthermore, if it is known (from some physical considerations) that a certain unknown is many times smaller than the rest of the unknowns, its decimal point may be shifted by using -10 or -100 in the corresponding row of the $-I$ matrix.

Obviously the "pivotal condensation" method may be easily incorporated into the described method, since it is a simple matter to select the row with the largest leading coefficient and use it as a pivot in the reduction process.

Computation Time Considerations. - The solution of a single set of equations of the form $(10 \times 10 \times 1)$ requires four hours when performed separately. It is quite evident that a multiple set of equations would require less time.

A set of 10 equations with 10 unknowns and multiple right hand sides, e.g., a set involving the same $A$ matrix but 14 columns in the $B$ matrix, $(10 \times 10 \times 14)$ requires ten hours, when a single set is computed. Again it is true that concurrent reduction of several sets will reduce the computation time.

The inversion of a single matrix of ten equations with ten unknowns requires eight hours. Again this time can be reduced by concurrent inversion of several matrices.

FrANK M. VERZUH

Mass. Inst. Technology

$1 \mathrm{~J}$. von Neumann, \& H. H. Goldstine, "Numerical inversion of matrices of high order," Amer. Math. Soc., Bull., v. 53, 1947, p. 1021-1099.

2 A. M. Turing, "Rounding-off errors in matrix processes," Quart. Jn. Mech. Appl. Math., v. 1, 1948, p. 286-308.

${ }^{3}$ L. Fox, H. D. Huskey, \& J. H. Wilkinson, "Notes on the solution of algebraic linear equations," Quart. Jn. Mech. Appl. Math., v. 1, 1948, p. 149-173.

${ }^{4}$ H. F. Mitchell, "Inversion of a matrix of order 38," MTAC, v. 3, p. 161-166, 1948.

5 Any person interested in obtaining information about the wiring diagrams used in this procedure should write directly to the author.

\section{RECENT MATHEMATICAL TABLES}

628[A].-H. E. Merritt, Gear Trains including a Brocot Table of Decimal Equivalents and a Table of Factors of all useful Numbers up to 200000. London, Pitman, 1947, viii, 178 p. $13.5 \times 21.4 \mathrm{~cm}$. Compare $M T A C$, v. 1, p. 21-23, 66-67, 91-92, 100, 132.

T. I. "The factor table," p. 15-54, contains the factors of the 4032 numbers $<200000$, having prime factors not less than 7 and not more than 127, the largest convenient tooth number in a change-gear train. T. II. "Brocot table," p. 65-95, 6D; the numerator of the fractions is $\leqslant 99$ and the denominator $\leqslant 100$; there is a column of exact remainders for each division. 